descriptive part is masked, and the student's mind left in a maze with the discussion of conflicting hypotheses. The latter half of the volume, on the anatomy of the arteries, is the best bit of real descriptive work in the book, but according to modern notions fails from the want of detailed relations. Many actual mistakes occur. He makes the superior laryngeal artery enter between the cricoid and thyroid cartilages, and brings the greater sciatic nerve over the pyriformis. Here the second edition ends, but to the third the author's brother Charles has added chapters on the remaining. parts of the anatomy. These consist of a very brief description of the brain and a still more insufficient account of the peripheral nerves, which in this edition are placed at the end of the second volume.

\section{Sperdal Senses.}

In the third volume, also by Charles Bell, the organs of sense are first treated, beginning with a psschological disquisition on sense perception, and a sketch of the principles of cptics, which occupy 25 pages. Then follow about 100 pages on the anatomy of the eye, of which more than onethird is taken up with digressions on the seat of vision, squinting, nyctalopia, etc. The anatomy of the ear is treated in much the same way, but here the description is padded out with accounts of the ear in the lobster and other animals, with details concerning diseases of the tympanum, and on the sense of hearing.

Abdominal Viscera.

After the senses come the abdominal viscera, treated more physiologically than anatomically, and he sarcastically criticises those who attempt to reduce physiology to a physical process. "Let us be satisfied with knowing a little. The life residing in the gland is an agent controlling the affinities."

The illustrations absent in the first volume, few in the second, are more numerous in the later third, but are of very little use in rendering the text intelligible. The student whose knowledge was derived from such a textbook might be amused with the destruction of effete theories, but would have, except in the article of arterial branchings, a poor acquaintance with human structure.

The century has taught us that anatomy is nothing if not definite; that anatomical illustrations for the use of students must be diagrammatised ; and that the real nexus whereby the discrete facts may be best strung together is morphological relationship. It has been said that human anatomy is a worked-out field; the comparison of old textbooks with those of the present day is the best refutation of this charge, which is based on igmorance, and it is by no means improbable that those who see the end of another century will be able to record advances both in knowledge and method even greater than those that have taken place in the last hundred years.

\section{和hysiolong in 1800 .}

BY HENRY POWER, M.B., F.R.C.S.,

Professor of Physiology Royal Veterinary College; Consulting Ophthalmic Surgeon St. Bartholomew's Hospital.

Physiorogy as a separate study can hardly be said to have existed in this country at the commencement of the present century. In books and in lectures alike it was united with, and considered a part either of medicine under the general term "Institutes of Medicine," or of anatomy. In the great schools of medicine of the Metropolis they were not divided until the first thirty years had passed away.
The University of London set the example, which was soon followed at Guy's Hospital. In an advertisement of 1833 the statement is made that lectures at this hospital were the only special lectures on physiology delivered in London, excepting the course at the London University, then under the charge of Dr. Sharpey. At Guy's the curious practice existed of confiding the teaching of physiology to the lecturer on midwifery, and Dr. Haighton and Dr. Blundell successively held the two offices. In 1846 anatomy and physiology having been in the interval taught simultaneously by Bransby Cooper, the two subjects were once more separated, and the lectureship on physiology was entrusted to Dr. (afterwards Sir William) Gull. Soon after, certain evenings in the week were devoted to instruction in microscopic work, and Mr. Birkett, who still survives to witness the immense development of the subject he taught in its infancy, was appointed the teacher. Histology was associated with physiology when Dr. Pavy succeeded to the chair in 1857, and classes in practical physiology, at which the use of the microscope was taught, were first instituted at this school in $187 \mathrm{r}$. The process of the separation of physiology from anatomy and the introduction of histology followed a similar course in other schools.

The extraordinarily rapid development of physiology is partly owing to the great advances that have been made in chemistry and physics, the sciences on which it rests, and partly to the progress effected in the means of observation. The balance and the methods of chemistry, the microscope and the methods of the histologist, have introduced a degree of precision into modern research unattainable by the older inquirers, who trusted to the unaided senses and have enabled observations to be made revealing the internal processes of the economy almost as astonishing as those which result from the application of the Roentgen rays in displaying the form and position of the bones of the living man.

The tendency of modern research is to show that all those acts and phenomena which were formerly regarded as the results of the operation of a special force, the vital force, are to be explained by the ordinary laws of physics and chemistry. There are many processes that are still enigmatical. We do not know the exact chemical changes that precede the evolution of hydrochloric acid in the gastric juice, nor the mode in which impulses are propagated along nerves, but it is presumed that in both instances intelligible, though perhaps not thoroughly comprehended, molecular changes take place, and that they are certainly not due to the supposititious "anima," or vital spark, which the older writers so readily accepted.

The physiological works published in the eighteenth century were few and unimportant, and were chiefly written in Latin or German. The monumental treatise of Baron Haller, the Elementa Physiologia Corporis Humani, appeared in eight volumes between the years 1757 and 1766. He had already, as early as 1747, sent forth a small book, entitled Prima Linea Physiologice, which was a precursor as well as a condensation of his larger work, and translations were immediately made into French and English. In 1786 a translation was published under the auspices of Dr. Cullen, the well-known Professor of Medlcine in Edinburgh. This book, with notes by Wrisberg, constituted almost the only physiological work in the hands of the student in this country, whilst the original was used in most of the foreign universities. It represents the high-water mark of physiological knowledge at the close of the last century, and in considering the progress of physiology during the present century it may advantageously be taken as standpoint. 


\section{BLOOD.}

Taking the subject of the blood and circulation, it may be noted that the physical characters of the blood, its coagulability, and the morphological elements it contains, were fairly well known, owing to the observations of Malpighi, Leeuwenhoek, and Jurin abroad, and those of Hewson and Hunter in this country. It is remarkable that no separate section is devoted in Haller's Prima Linea to an account of the blood, the littlethat is said of it being given under the head of "Secretion." The corpuscles were held to be globular, but the estimates of their size were remarkably correct considering the instruments used. Blumenbach gave their diameter as $\frac{1}{3 \frac{1}{3} \sigma}$ inch, and

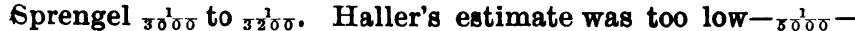
but the method he adopted was ingenious, for he compared their size with that of the scales on the wings of a butterfly, which, being arranged in rows, permitted the number in an easily measurable space to be counted. The composition of the blood, the existence of hæmoglobin with its special affinity for oxygen, the origin of the blood corpuscles, their numbers, the mobility and diapedesis of the white corpuscles, the absorption bands the blood presents under spectrum ansIysis were all unknown, and the cause of the coagulation of the blood, which, however, is even now a subject of discussion, is wholly ignored.

\section{Circulation.}

The fundamental facts in regard to the action of the heart and the circulation of the blood were established by Harvey in the seventeenth century, and the principal addition made to his conclusions in the eighteenth were founded on the classical and extremely ingenious experiments of Hales on the pressure of the blood in the vessels. The importance of a correct appreciation of the causes of the sounds of the heart and their relation to its movements in medicine led to many researches being undertaken both in this country and abroad at the commencement of the present century. It was not surprising in view of the many difficulties attending the experiments that the conclusions arrived at were in some instances conflicting, and the order of the movements of the heart and of its divisions could scarcely be regarded as settled until the introduction of the graphic method in the hands of Volckmann, Vierordt, Ludwig, and Marey. The employment of the mercurial manometer by Poiseville in place of the long and'cumbrous glass tube of Hales enabled the blood pressure in esch vessel and in different animals to be easily ascertained, and the cleverly-devised double tubular sound ending in an elastic bag devised by Chauveau, and introduced through the vena cava and auricle into the right ventricle, permitted the pressure of the blood even in those not easily accessible cavities to be registered with comparative facility. The remarkable observation of the brothers Weber in 1845 on the restraining influence of the vagi on the heart opened up a ne $\pi$ chapter in physiology, and the phenomena of inhibition, as the effect is named, have been shown to constitute an important function of the nervous system in many other instances. It led to careful examination of the influence of other nerves on the action of the heart, and the origin and course of the accelerating and inhibitory cardiac nerves are now well known. The discovery by Cyon of the depressor nerve, which influences the action of the heart by acting as a vaso-dilator of the vessels of the abdominal viscera, was also of great value. The ephygmograph, by which the number and form of the pulse waves can be accurately shown, though valuable in some instances, presents difficulties in its clinical application that has prevented its general introduction into medical practice. The structure of the heart and vessels was first thoroughly elucidated by the careful microscopic observations of Kölliker, and various instruments have been devised by Volckmann, Ludwig, and others to determine the rapidity of the pulse wave and of the flow of the blood.

Secretron.

The knowledge of protoplasm and of its immense importance being unknown, perhaps the chapter in physiology in which the teaching was most foggy was that dealing with the secretions of the various glands. Withdrawn from observation by their concealed position, the subjects of innumerable fanciful and often absurd speculations, misunderstood by reason of the imperfect chemical knowledge of the day, such glands as the liver and pancreas with their secretions were riddles hard to read by Haller and his contemporaries. Their conclusions were vitiated by two errors. First, that the secretions existed preformed in the blood, and, secondly, that the blood vessels and the ducts of the glands were in their finest ramifications continuous with each other. Thus Haller observes that "there are many argaments to persuade us that the liver is a mere vascular fabric, whence the bile distils immediately from the extremities of the porta into the pori biliarii or roots of the biliary ducts without passing any cells or follicles by the way."

Elsewhere, after pointing out that the blood contained coagulating serum, water, viscid mucus, and oil, he maintains that the blood vessels entering the glands represent strainers the length, size, and [form of which determine both the nature and the escape of the particular constituent of each secretion. The differences in the quality of the secretion depended, in his view, upon the position its particles occupied in the blood stream. Fat and marrow were believed to be deposited without the intervention of glands from the small mouths of the minute arteries into the cellular substance; the secretion of the various forms of sebaceous glands, and of milk, was due to the separation of the oily portions of the blood, and the mucus of the surface of the intestine and genito-urinary tract to the separation of the mucus previously existing in the free state in the blood.

The secretions of the thinner juices were considered to be made not from sanguineous but from smaller pellucid arteries arising from the former, to the secerning mouths of which not only no red blood, but no serum, fat, or other gross material could have admittance. It is in this way "that the more thin and pure humours" -those of the eye, for example- " must necessarily be sepa. rated." Nor was less importance attached to the angles at which the smaller vessels were given off from the trunks; difffrent secretions being formed according to whether the mode of ramification of the arteries was, as in the spleen wisp-like, or as in the intestines pencil-like, or like serpents as in the kidneys or in the form of stellæ as in the liver or in a "radiated circle" as in the vivea, or like a lock of hair curled up into the form of a button as in the testis.

Haller well knew how to cover ignorance by high-sounding phrases, and the following paragraph may be quoted as a good example of darkening counsel by words. Speaking of the bile he says: "It takes in the saponaceous water of the stomach by the absorbing veins together with the subfetid alkalescent vapours of the abdomen, which transpire through the whole surface of the intestines, stomach, omentum, liver, spleen, and mesentery, which are absorbed again by the veins as we know by incontestable experiments of anatomy ; and, finally, the alkalescent semi-putrid acrimonious humidity from the fæces, while they continue to thicken in the large intestines, which is taken up by the internal hæmorrhoidal veins, from 
whence that bitterness, alkalescent, and putrescent disposition of the bile is derived."

It is curious to find that as late as 1823 Adelon in his physiology declaring without hesitation that the blood vessels and the ducts of the glands are continuous without any intermediate organ, the former discharging by their capillary extremities the fluid which is secreted from the blood, only since the blood can no longer reach this point it is no longer called a blood vessel but an exhalant vessel. It was Müller who in 1830 first demonstrated that these two systems of tubes were not continuous. Dutrochet was amongst the first to recognise the importance of the cell, and its structure was studied by Purkinje, Schleiden, Schwann, Henle, Huxley, and Bowman, and in later years by Flemming, Strasbürger, Leydig, Frommann, Hertwig, and many others, with wonderful and far-reaching results affecting every department of physio!ogy.

The labours of $C$. Bernard, Pavy, and a host of other observers have thrown a flood of light on the functions of the liver and have demonstrated that whilst there may be a conspicuous outward or excrementitial result of the activity of the gland, as in the case of the bile, which has its own pur. poses to fulfil in the economy, there may also be a not less important internal or excrementitial product, as glycogen, which is entirely concealed from superficial observation, whilst there are organs like the thyroid or adrenals that yield no visible secretion yet exercise a considerable influence on the general nutrition of the body by discharging the products of their activity into the blood. The structure of the kidney and the composition of the urine were not thoroughly understood until the masterly researches of Bowman demonstrated the relation of the blood vessels to the urinary tubuli which were followed by those of Henle on the epithelium of

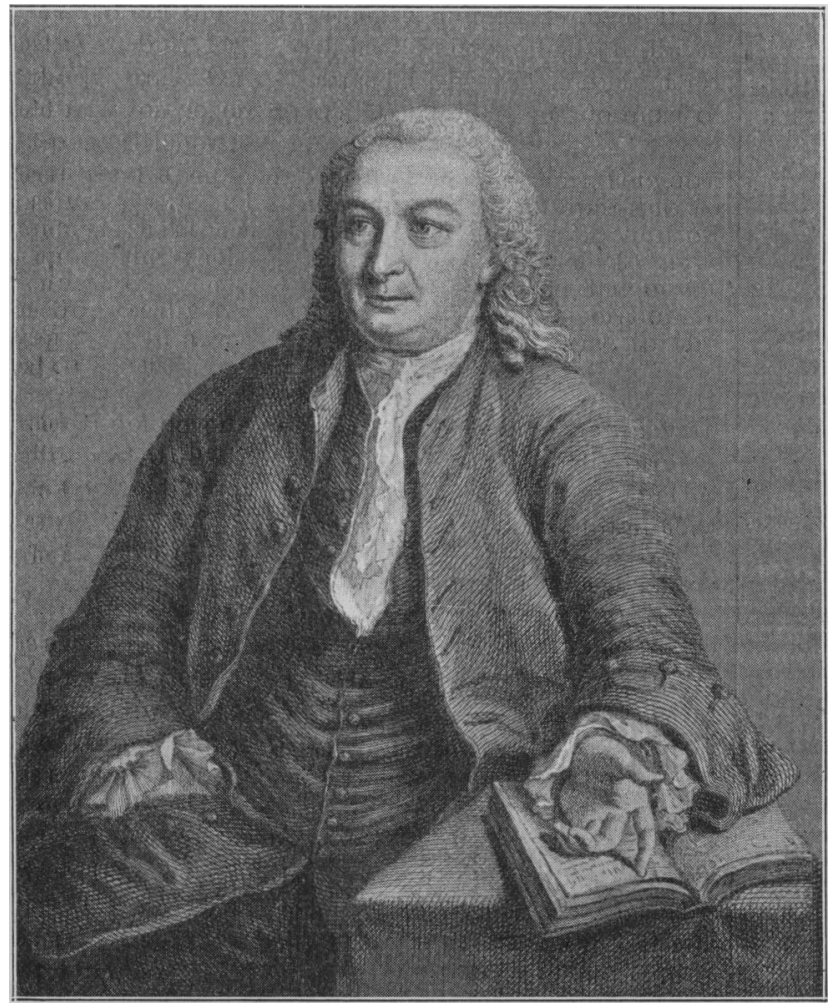

ALBRECBT VON HALLER.

Physiologist, Botanist, Poet. Born, r708; died, 1777 thought that in the lung substance the small arteries exhaled a plentiful vapour into the cells, whilst the veins absorbed a watery vapour from the same cells. Hence a thin solution of wax could be made to pass from the arteries into the air cells, and from thence to the bronchi; or from the veins into the same spaces, and so to the bronchi; or lastly, from the arteries into the veins, and vice versâ, the air cells being thus regarded as the common meeting place and seat of free communication of the minutest arteries, veins, and air tubes. Yet he elsewhere says that in the lungs the "cold air very nearly touches the blood." The unsatisfactory condition of chemistry in the period antecedent to Cavendish and Priestley rendered it impossible to give an intelligible account of the changes occurring in respired air.

So little was the entrance of air into the blood by chemical affinity understood, that it is stated that "no elastic air is here received into the blood as is demonstrated from the impossibility of forcing air into blood if it retains its elasticity, from the inutility of its reception, if the spring of it should be lost, into the blood; from the perfect immutability of the blood by cold: from the minuteness of the inhaling vessels, with the mucus that perpetually lines the sides of the vesicles in the lungs, to which add the nature of the elastic air self, which is very unapt to pass through capillary ves sels, with a repulsion of it by water that hinders it from passing paper, linen cloth, or skins that are wetted by water." It is thought necessary to assure the reader that the air being driven into the windpipe never passes the heart, or whenever it does it is forced thither by violence. The effects of the passage of the blood through the lungs on the temperature of the blood was very ob. scurely stated. It was felt that the warming of the expired air and evaporation of the watery vapour must coo the tubuli. The composition of the urine, to which Haller devotes only a short paragraph, is now accurately known through the labours of innumerable observers in all countries. The influence of the nervous system on the glands has been clearly shown in the case of the salivary, sweat, lachrymal and other instances.

\section{Respiration.}

The mechanism of respiration was well understood in Haller's time. More than a quarter of a century was to elapse before the normal and abnormal sounds of the respiratory acts were to be rendered familiar to all by $L$ ännec through the application of the stethoscope. The chemical processes involved in those acts had just been elucidated by the genius of Lavoisier, but his clear exposition of them had not as yet gained entrance into the texiliooks. Haller seems to have blood; but on the other hand, since arterial blood is warmer than venous, the blood must acquire its heat in the lungs, and in this error even Lavoisier participated. After the above quotation, in which phrases are used only to cover ignorance, let us give the simple and pregnant, but at the time he wrote them perfectly novel, expressions of Lavoisier, about 1776. "Of two things," he says, "one must occur : either, the portion of oxygen contained in the air is converted into carbonic acid gas in traversing the lungs, or an exchange of gases takes place in this organ. On the one side oxygen is absorbed, and, on the other, the lung restores a quantity of carbonic acid gas of nearly equal volume." He subsequently read a memoir, in which he maintained that a combustion or oxidation of hydrogen took place in the lungs. Of course 
nothing was known of the process of internal respiration, or the exchange of gases between the tissues and the blood.

\section{animal Heat.}

The imperfect knowledge of chemistry led to the wildest guesses at the cause of the high and uniform temperature of the animal body. By many the heart was regarded as the source of animal 'heat, the blood there undergolng a kind of fermentation ; by others it was attributed to the friction of the blood corpuscles against the walls of the blood vessels, and by others again to some obscure influence of the nervous system. The extremely ingenious and careful experiments of Lavoisier led physiologists to juster views. He was even able by means of his calorimeter to ascertain the total quantity produced. At the time the Prima Linea were written, though nearly ten years had elapsed from the time when Lavoisier's views were published, they had not received general acceptance, or at-least were not taught. Haller's views were not clear upon the subject, and he deals with animal heat very slightly. The chief error of Lavoisier was the conviction that animal heat was generated in the lungs themselves instead of in the tissues of the body at large.

The observations of Hunter and of Dr. John Davy on the temperature of different races of man in various climates, and subsequently of Newport on the temperature of bees, and of Valentin on man, were highly suggestive and in structive. By the use of a thermo-electric apparatus Nobili and Nulloin were able to recognise very slight changes of temperatures. The influence of functional activity on the local temperatures was shown by Ludwig in the case of the salivary glands, and the action of the sympathetic on the vaso-motor system, and its secondary action on temperature by Brown-Sequard and Schiff. Of late years the results of the careful experiments of Frankland, Ranke, Hirn, Helmholtz, Rubner, and others on the heat equivalents of different kinds of food and upon the seats and amount of heat production have shown the uses of applied physiology in providing appropriate and economical food for sailors, soldiers, prisoners, and other bodies of men, both when at rest and when doing work, and also by furnishing a measure of tissue consumption constituting a most valuable aid in the prognosis and treatment of febrile diseases.

Such inquiries also showed and furnished an explanation of the correlation of hibernation and such phenomena as the development of fur and change of plumage according to season in animals with variations of temperature.

Digestion.

Digestion, the details of which occupy so large a space in modern textbooks, was very imperfectly understood. The opinions of Pitcairn who thought that the muscular force of the stomach was equal to 117,088 pounds weight, and was supplemented by an equal force derived from the diaphragm and abdominal muscles; as well as that of Dr. Drake, that digestion is performed in the stomach, as in a Papin's digestor ; and that of Cheselden, who held that digestion is no other than corruption or putrefaction of our food, and is performed by a menstruum which is chiefly saliva, were discredited after the experiments of Reaumur and Spallanzani, but it was reserved for Dr. Prout in 1824 to show that the gastric juice contained hydrochloric acid, and for Schwann in 1836 to isolate pepsin. Haller maintained that in fasting the stomach contained a considerable quantity of a limpid fluid or humour resembling saliva, but more mucous, and that the sensation of hunger proceeds from the attrition of the sensitive folds of the lining membrane of the stomach. His account of digestion is as follows: "The aliments taken into the stomach begin to corrupt or change into a nauseous liquid, often ascescent or putrescent, though this is less frequent in mankind from our use of bread and salt, or it may be rancescent, as appears from the flatus and other matters eructated, often of a most fœtid, caustic, and inflammatory nature." From. which we may conclude, if the Baron described his own symptoms, that either his food was not very wholesome or his digestion was very bad. He ends with the remark that there is, therefore, no particular kind of ferment in the stomach "from which the design of Nature, the disposition of the stomach and its use, are all very remote."

The chemistry of the digestive processes, almost entirely unknown, as we have seen in Haller's time, has of late years attracted the attention of a multitude of observers, partly owing to the ease with which the fluids can be obtained in a nearly pure state by the formation of fistulæ, and partly from the interest which is excited by the attempt to follow the steps effecting the conversion of non-living into living matter. Tiedemann and Gmelin, Liebig, Strecker, Majendie, Kühne, Bernard, Leuret and Lassaigne. Liebig, Bernard, Chauveau, Colin and Kühne, and Pavy suggest themselves at once as the names of a few only of those who have followed generally the changes of the food in the stomach and intestines, or who have studied the action of the fluids poured into the alimentary canal by the salivary, gastric, pancreatic, hepatic, and intestinal glands. But, perhaps, none made such solid contributions to our knowledge on this subject as Dr. Beaumont, who about 1834 continued his observations for more than a year on a young Canadian who had a gastric fistula from a gunshot wound permitting the digestion of numerous articles of food to be carefully watched. The general result has been to rectify many errors into which the older physiologists fell, 'and to place our knowledge of the processes of digestion on a sound basis. The earlier changes that the different kinds of food undergo, whether oily, starchy, or albuminous, are now fairly well known, as well as the mode in which they are absorbed, and the channels by which they pass into the blood, though much remains to be done before the assimilation of food can be regarded as thoroughly understood.

The account of the pancreas and of its secretion, given by Haller, constitutes a measure of the advances that have been made in our knowledge of that gland. No account of its structure is given, but the pancreatic juice he describes as watery, insipid, thin, neither acid nor alkaline, and as the gland is three times larger than the salivary glands, the quantity of the fluid it discharges must, he thinks, be considerably greater. Its expulsion, is due partly to the force of the circulating blood, and partly to an alternate pressure from the incumbent and surrounding viscera. Its use is to dilute the viscid cystic juice, to mitigate its acrimony, and mix it with the food. It fulfils every office of the saliva. It need hardly be said that now, after the researches of Claude Bernard, that secretion would be described in very different terms, as highly alkaline, ropy, small in quantity, and acting on starches, oils, and proteids, through the action of different ferments.

\section{Neavous SYstru.}

But perhaps no department of physiology has undergone greater and more striking changes than that of the nervous system. It has been attacked on many sides. The scalpel has followed the course of the fibres with the nicest care; all 
the methods known to the histologist have been applied to it. Fragments have been separated, hardened immediately upon their removal from the body, embedded in supporting material, cut with sharp knives into the finest sections, stained with one, two, three, or more colouring ogents, in acid, neutral, or alkaline solution, affecting differently the various elements; mounted in transparent balsams and examined with microscopic lenses that magnify a thousand times or more the apparent size of objects. Based on the fact that the portion of a nerve fibre remaining continuous with the nerve cell from which it springs preserves its histological characters when the nerve is divided, whilst the distal portion undergoes very obvious changes, fatal to the performance of its function, what have been termed degeneration experiments have been performed by Waller and others ; and by the aid of these the course of fibres and the functions of different parts have been determined. The distinctive characters of the anterior and posterior roots of the spinal nerves were ascertained independently of each other by Sir Charles Bell and Majendie. The theory of reflex action was developed by Dr. Marshall Hall. The structure of the spinal cord was carefully investigated by Lockhart Clarke and Schroeder Van der Kolk. Their investigations have been largely extended; and the columns of the cord, in which definite bundles of nerves are united, have been distinguished by Goll and Burdach and Turck and Gowers. This knowledge has proved of great importance in localising disease.

The whole subjest of the functions of the brain was of course very superficially known. The functions of the different parts which were anatomically described with sufficient precision, could not even be guessed at as long as the brain and cerebellum were supposed to be insensitive to tactile and other impressions. The high sensibility of the dura mater led to curious ideas in regard to its functions. The seat of the soul was discussed and placed successively in the pineal body, the corpus callosum, the corpora quadrigemina, and even in the liquid contained in the ventricles of the brain; by Haller himself it was more sensibly held to be situated in the crura of the medulla, corpora striata, thalami, pons, medulla oblongata, and cerebellum, and the origins of all the nerves. Nevertheless, the mistaken notion of Malpighi that the cortical substance of the brain is glandlike in structure, and that the fluid secreted is poured into the tubes of the medullary portion, which constitute excretory ducts, was still held.

The nerves were seen to be composed of fibres, and these fibres were known to be continuous with the fibres of the brain. Some indeed thought they were solid threads which, when excited, vibrated and conducted the particular impressions to the brain, but Haller notices their softness, their want of elasticity, and absence of tension, and after adducing various arguments to show that the nerves could not act as vibrating cords, concludes with this remark: "Therefore, upon the whole, it geems to be certain that from the vessels of the cortex a liquor is separated into the hollow pipes of the medulla which are continuous with the small tubes of the nerves, even to their soft pulpy extremities, so as to be the cause both of sense and motion. But there will be a twofold motion in that humour, the one slow and constant from the heart, the other not continual but exceedingly swift, which is excited either by sense or any other cause of motion arising in the brain." Jastly, as the fluid could not be constantly pouring into the nerves, and not overflow, he thinks that it probably exhales through the cutaneous nerves; possibly some may be resorbed and may exhale into the various cavities of the body, as the stomach and intestines.

Of the existence as well as of the advantages of the decussation of many nerves Haller was well aware.

The observations of Hitzig and Ferrier-that stimulation of certain convolutions of the brain was followed by responsive action of various muscles, sufficiently constant to be predicted with tolerable certainty-opened up a new field, with the result, to some extent anticipated by Broca-who indicated the third left frontal convolution as specially dedicated to speech-that certain areas of the cortical substance had as their function the reception of the impressions of the several sonses, whilst other convolutions presided over or originated movements of various muscles; and at the present time it is accepted by all physiologists that the occipital lobes are the psychical centres of vision, the temporo-sphenoidal lobes those of hearing and smell, and that the ascending parietal and ascending frontal convolutions proceeding from above downwards are related to the muscles of leg, arm, and face. The functions of the cerebellum still remain to be elucidated.

Some progress had been made by 1800 in the physiology of the special senses, especially of the eye, but perhaps no parts of the body have been of late years investigated during the nineteenth century with such extraordinary diligence and success as these organs. This is evidenced both by the advances that have been made on the purely theoretical side as well as by the improvement in practice that has taken place in the diagnosis and treatment of their diseases by the application of such instruments as the ophthalmoscope.

The subject of muscular contraction has been also pursued with great perseverance. Haller recognised in muscle elas ticity, and irritability, the vis insita, or, more correctly speaking, contractility and sensibility, but little was known either of its structure or properties. Buwman gave the first full and accurate description of its histology. Krause, Dobie, and others followed. Its composition was studied by Kühne, Brücke, and others, and many articles have been written on the sources of muscular energy, the metabolism that takes place in muscle during rest and fasting, and in full dietend work. The form and rapidity of the wave of contraction has been investigated with special instruments. The phenomena of tetanus and of rigor mortis have been examined, and the action of muscles in various positions of the body and in various movements have been followed out.

\section{EMBryology.}

Of embryology there is no space to speak; it is enough to say that it constitutes one of the most vigorous offshoots of the parent stem, and that many observers are engaged in the problems it offers to the naturalist.

\section{Applications to Hygikne.}

A secondary but by no means unimportant result of the study of physiology has been its practical application to the affairs of daily life. Applied physiology points out the necessity of food that shall be abundant and wholesome, of free supply of water for drink and for ablutions, regular exercise in the open air, good ventilation in houses, appropriate intellectual training for the child, and avoidance of excessive mental and bodily exertion for the adult. The nation that will follow the dictates of sound physiology will have the best developed and healthiest men and women for its constituent units, and will have the ferest hospitals, asylums, and prisons. Disease and premature decay are in a large number of instances the result of the neglect or opposition to the rules of physiology intelligently applied. 